\title{
M. Reimer, P. Getimis and H. Blotevogel: Spatial planning systems and practices in Europe: a comparative perspective on continuity and changes
}

\author{
Routledge, London, 2014, 336 pp, ISBN: 978-0-415-72723-5
}

\section{Thomas Hartmann • Tejo Spit}

Published online: 15 October 2014

(C) Springer Science+Business Media Dordrecht 2014

There are numerous comparative studies on European spatial planning systems (CEC 1997; Knieling and Othengrafen 2009; Newman and Thornley 1996). Why do we need one more, and how does this one differ from the rest?

This book compares planning systems in twelve European countries: Belgium (Flanders), the Czech Republic, Denmark, Finland, France, Germany, Greece, Italy, Poland, The Netherlands, Turkey, and the UK. Each country is presented in a separate chapter. The authorship comprises a mixture not only of professors, but also of planning professionals and young academics. Overall, the quality of the chapters on planning systems is very high. Some contributions are stronger in terms of theory (such as the description of the Greek system); others focus more on a case-study approach. The description of each planning system follows a similar structure: a short introduction reviewing the context of the various systems, a brief elaboration on the basic features of the planning system in question, an identification of pressing problems and challenges, descriptions of "dimensions and directions" of changes, and a conclusion. In the first chapter of the book, the editors introduce this analytical framework. In the conclusion of the book, the editors reflect on the diversity of the different spatial planning systems. This structure is very much in line with other comparisons of planning systems.

Reimer and his colleagues claim that their book differs because they pursue a different approach than that of the "Newman and Thornley tradition" (Reimer et al. 2014, p. 3). This tradition of comparative research focuses on legal-administrative framework conditions. Reimer and his colleagues criticize this tradition because it overlooks many issues that characterize spatial planning in practice. However, most chapters in this book place a strong focus on legal-administrative issues in the planning systems: institutions, laws, and

T. Hartmann $(\bowtie) \cdot$ T. Spit

Department of Human Geography and Planning, Faculty of Geosciences, Utrecht University, Utrecht, The Netherlands e-mail: t.hartmann@uu.nl

T. Spit

e-mail: T.J.M.Spit@uu.nl 
structures. In this respect, the book is a prime example of planning-led thinking, and it actually does follow the "Newman and Thornley tradition." The editors of the book seem to avoid this notion; nevertheless, such a perspective can be very valuable. Within academic circles, there is a growing interest in international comparisons and discussions on the legal issues related to planning. ${ }^{1}$ So, the strong focus on the institutional aspects of planning systems is, in fact, one of the strengths of this publication.

There are at least two more reasons why researchers in spatial planning should have this book on their shelf. The first reason is the book's analytical framework. According to the blurb on the back, the book "develops a systematic and methodological framework for analyzing changes in planning systems throughout Europe." This framework focuses on five particular "dimensions of change" in spatial planning. These five dimensions are scope and objectives, modes and tools, scales, actors and networks, and policy and planning styles. This is a very comprehensive and structured way of comparing planning systems, and it makes the direct comparison between the countries very accessible. On a critical note, the reason for choosing these five particular dimensions is not well explained (although, institutionally, they seem to cover all important aspects). Aside from this critique, the book's methodological framework is very useful. Because the framework parameters are well described in the introduction, they can be used as a blueprint for analyzing and comparing further planning systems that have not been included in the book.

This leads to the second strength of this book: it covers a broad scope of planning systems. The book reviews planning traditions from all categories of the EU Compendium of Spatial Planning Systems and Policies (CEC 1997). This compendium distinguishes four types of planning traditions, namely regional-economic, urbanism, comprehensive/integrated, and land-use planning. The editors take care that, for each of the four types, at least two planning systems are represented in the book. This makes the selection of planning systems quite complete and comprehensive. Unfortunately, these categories are applied in the book without supporting explanation or justification. Beyond this drawback, however, the selection of countries is remarkable in many respects in terms of its diversity and coverage of different systems. The value of the book lies in its structured comparison of these various planning systems.

As a compliment to the authors, it should be mentioned that this book is well written and very accessible. It is useful for academics because it provides an interesting method and a structured comparison of different planning systems. Moreover, the book is important for practitioners and policymakers, as it provides needed information and enriches reflection toward one's own system, thereby encouraging innovation and adaptation.

On a final note, it is worth mentioning that the German Academy for Spatial Research and Planning (ARL) supported the development of this book. This institution is a German network of planning experts and academics. The institution is traditionally more oriented toward Germany. In its support of this book, it is encouraging to see the ARL entering the international planning debate. Hopefully this international focus will continue, as it will enrich the academic community on spatial planning and research.

1 The track on Planning, Law, and Property Rights at the planning congress AESOP was one of the largest in 2014. 


\section{References}

Commission of the European Communities (CEC). (1997). The EU compendium of spatial planning systems and policies. Luxembourg: European Communities.

Knieling, J., \& Othengrafen, F. (2009). Planning cultures in Europe: Decoding cultural phenomena in urban and regional planning. Farnham: Ashgate.

Newman, P., \& Thornley, A. (1996). Urban planning in Europe: International competition, national systems, and planning projects. London: Routledge. 